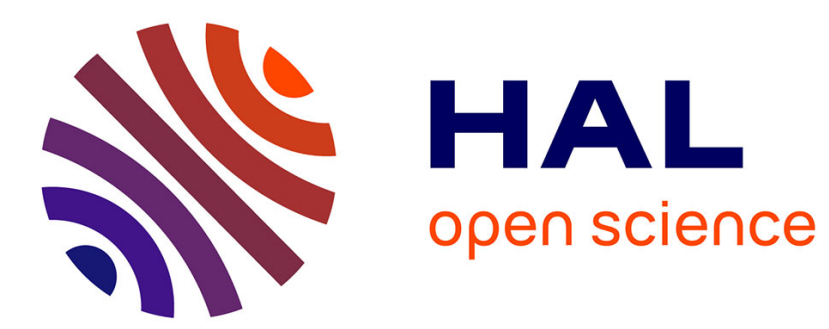

\title{
La clinique freudienne de l'entrée dans la psychose
}

Yohan Trichet

\section{To cite this version:}

Yohan Trichet. La clinique freudienne de l'entrée dans la psychose. Recherches en psychanalyse, 2011, 12 (2), pp.196 - 205. 10.3917/rep.012.0196 . hal-01728294

\section{HAL Id: hal-01728294 \\ https://hal.univ-rennes2.fr/hal-01728294}

Submitted on 10 Mar 2018

HAL is a multi-disciplinary open access archive for the deposit and dissemination of scientific research documents, whether they are published or not. The documents may come from teaching and research institutions in France or abroad, or from public or private research centers.
L'archive ouverte pluridisciplinaire HAL, est destinée au dépôt et à la diffusion de documents scientifiques de niveau recherche, publiés ou non, émanant des établissements d'enseignement et de recherche français ou étrangers, des laboratoires publics ou privés. 


\title{
LA CLINIQUE FREUDIENNE DE L'ENTRÉE DANS LA PSYCHOSE Yohan Trichet
}

\author{
Association Recherches en psychanalyse | «Recherches en psychanalyse »
}

2011/2 n $12 \mid$ pages 196 à 205

ISSN 1767-5448

Article disponible en ligne à l'adresse :

https://www.cairn.info/revue-recherches-en-psychanalyse-2011-2-page-196.htm

\section{Pour citer cet article :}

Yohan Trichet, «La clinique freudienne de l'entrée dans la psychose », Recherches en psychanalyse 2011/2 ( $\left.\mathrm{n}^{\circ} 12\right)$, p. 196-205.

DOI 10.3917/rep.012.0196

Distribution électronique Cairn.info pour Association Recherches en psychanalyse.

(C) Association Recherches en psychanalyse. Tous droits réservés pour tous pays.

La reproduction ou représentation de cet article, notamment par photocopie, n'est autorisée que dans les limites des conditions générales d'utilisation du site ou, le cas échéant, des conditions générales de la licence souscrite par votre établissement. Toute autre reproduction ou représentation, en tout ou partie, sous quelque forme et de quelque manière que ce soit, est interdite sauf accord préalable et écrit de l'éditeur, en dehors des cas prévus par la législation en vigueur en France. Il est précisé que son stockage dans une base de données est également interdit. 


\section{Recherches en Psychanalyse - Research in Psychoanalysis}

12 |2011- Psychanalyse, corps et Société

Psychoanalysis, the Bodv and Society

Penser la psychose

\section{La clinique freudienne de l'entrée dans la psychose}

The Freudian Clinic of the Onset of Psychosis

Yohan Trichet

\section{Résumé :}

L'auteur se propose d'examiner la genèse et l'évolution de la clinique freudienne de l'entrée dans la psychose, à partir des observations contenues dans le corpus freudien. II retrace le parcours conceptuel du fondateur de la psychanalyse, de ses premiers textes sur les psychonévroses (1894) à ses articles afférents à la seconde topique dans les années 20. II en ressort que Freud situe essentiellement ses recherches dans le champ des psychoses paranoïaques. Malgré son incessante volonté et ses nombreuses propositions spéculatives, Freud ne parvient pas à dégager le mécanisme spécifique de la psychose et de son éclosion.

\section{Abstract :}

The author aims to examine the genesis and evolution of the Freudian clinic of the onset of psychosis, based on the entire body of Freud's work. He follows the conceptual development of the founder of psychoanalysis, from his first texts about the psychoneuroses (1894) to the essays of the late 1920s, and shows that Freud's research mainly concerns the field of paranoiac psychosis and that despite his efforts and several speculative propositions, Freud fails to identify the specific mechanism of psychosis and of its triggering.

Mots-clefs : entrée dans la psychose, Freud, paranoïa, délire, Verwerfung

Keywords : onset of psychosis, Freud, paranoia, delusion, Verwerfung

\section{Plan :}

\section{Introduction}

1. Les psychonévroses de défense : le cas de la paranoïa

1.1. Les psychonévroses de défense (1894)

1.2. Le manuscrit $H(1895)$

1.3. Le manuscrit $\mathrm{K}$ (1896)

1.4. Les nouvelles psychonévroses de défense (1896)

2. Le Président Schreber (1911)

2.1. La période d'incubation

2.2. Les modes paranoïaques de contradiction du fantasme homosexuel

3. Un cas de paranoïa chez une jeune femme (1915)

4. Le cas d'un jeune médecin (1916-1917)

5. Un accès de jalousie délirante (1921)

6. L'entrée dans la psychose et la seconde topique freudienne

Conclusion : la quête freudienne 


\section{Introduction}

Freud n'a pas produit de théorie systématique de l'entrée dans la psychose, ne parvenant pas à formaliser ses investigations dans un concept original et spécifique, à l'instar du refoulement pour la névrose. ${ }^{1} \mathrm{~S}^{\prime}$ il consacra un article à la clinique de l'entrée dans la névrose intitulé Sur les types d'entrée dans la névrose ${ }^{2}$, il ne le fit pas pour la psychose. Néanmoins, l'intérêt du fondateur de la psychanalyse pour cette catastrophe subjective fut indéniable. II ne cessa de vouloir la rendre intelligible. Le corpus freudien contient, en effet, au moins sept cas cliniques d'entrée dans la psychose que nous allons présenter dans cet article.

\section{Les psychonévroses de défense : le cas de la paranoïa}

\subsection{Les psychonévroses de défense (1894)}

À l'encontre de la doctrine de la dégénérescence, Freud considère dès 1894 l'hystérie, l'obsession et la confusion hallucinatoire comme " trois formes de défense ". $^{3}$ Il les appréhende comme autant de variétés cliniques de défense du moi luttant contre une représentation inconciliable avec lui, au demeurant d'efficacité inégale. À cet égard, la confusion hallucinatoire est une

[...] espèce beaucoup plus énergique et efficace de défense [que les deux autres]. Elle consiste en ceci que le moi rejette [Verwift] la représentation insupportable en même temps que son affect et se comporte comme si la représentation n'était jamais

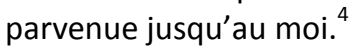

Il s'agit de la première occurrence de la Verwerfung freudienne qu'il appliquera plus tard à l'hystérie. ${ }^{5}$ II n'en fera donc pas un mécanisme inhérent à la psychose. En outre, il affirme que le contenu d'une confusion hallucinatoire "consiste précisément en la mise au premier plan de cette représentation qui était menacée par l'occasion déclenchante de la maladie $"{ }^{6}$ Freud dégage cette thèse de l'observation d'une jeune femme, amoureuse d'un jeune homme dont elle s'imagine être aimée en retour. Après quelques désillusions ayant engendré des symptômes de type hystérique survient un événement décisif : un jour, alors que ce jeune homme est convié à une fête au domicile familial de la jeune femme, il ne vient pas. Cette attente génère chez elle un état de tension psychique qui atteint son paroxysme à la fin de la journée :

Elle verse dans une confusion
hallucinatoire. Il est arrivé, elle entend sa
voix dans le jardin, elle court en chemise de
nuit pour l'accueillir. À partir de ce jour, elle
vit pendant deux mois dans un rêve heureux
dont le contenu est le suivant : il est là, il est
toujours près d'elle, tout est comme par le
passé. $^{7}$

Selon Freud, la représentation de l'absence de ce jeune homme a subi une Verwerfung. II en déduit que le processus psychotique peut être conçu ainsi :

Le moi s'arrache à la représentation
inconciliable, mais celle-ci est
inséparablement attachée à un fragment de
la réalité, si bien que le moi, en
accomplissant cette action, s'est séparé
aussi, en totalité ou en partie, de la réalité.

En appréhendant I'hallucination comme une satisfaction du moi générée par la forclusion (Verwerfung) d'une représentation inconciliable avec lui, Freud renouvelle la conception traditionnelle de l'entrée dans la psychose.

\subsection{Le manuscrit $H$ (1895)}

Dans ce manuscrit, Freud intègre la paranoïa dans le groupe des psychonévroses et envisage son éclosion comme l'activation d'une défense spécifique devant une situation intolérable au sujet. ${ }^{9}$ II fait valoir la notion de prédisposition psychique à la paranoïa et s'attache dès lors à en éclairer le mécanisme. Dans ce sens, Freud présente le cas d'une femme d'une trentaine d'années souffrant d'un délire de persécution. Quelques années auparavant, elle a relaté à sa sœur aînée une scène qui s'est produite chez 
elles : un jeune homme qu'elles hébergeaient lui a placé sa verge dans la main. S'ensuit l'éclosion d'un délire :

Les voisines la plaignaient parce qu'elle était un laissé-pour-compte et qu'elle attendait le retour de cet homme. On lui faisait sans cesse des allusions de ce genre, on jasait à propos de cette histoire, etc. ${ }^{10}$

Constatant chez sa patiente la négation de cette scène traumatique, Freud s'interroge sur la singularité de cette défense paranoïaque :

Elle tentait d'échapper au reproche d'être " une vilaine femme ». Mais ce reproche lui vint du dehors et ainsi le contenu réel resta intact alors que l'emplacement de toute la chose changea. Le reproche intérieur fut repoussé en dehors: les gens disaient ce qu'elle se serait, sans cela, dit à ellemême. $^{11}$

Centré sur le mécanisme générant le délire, Freud allègue que

[...] le but de la paranoïa est donc de se défendre d'une représentation inconciliable avec le moi, en projetant son contenu dans le monde extérieur. ${ }^{12}$

Il s'interroge alors sur le mécanisme de la projection et ses modes opératoires. Freud répond qu'il s'agit d'un mésusage pathologique du mécanisme de projection utilisé à visée défensive. Quand le sujet a conscience du contenu de ses idées et affects mais aussi de leurs origines, il est peu étonné qu'un autre puisse les deviner. II s'agit là, selon Freud, du fonctionnement normal du mécanisme de la projection. En revanche, lorsque le sujet oublie l'origine intérieure de ses propres idées et affects, car intolérables, il attribue à un autre leur contenu : c'est là le mésusage paranoïaque de la projection. Puis, Freud se demande si ce mécanisme fonctionne à l'identique dans d'autres cas de paranoïa. Il estime que d'une manière générale

[...] la ténacité avec laquelle le sujet s'accroche à son idée délirante est égale à celle qu'il déploie pour chasser hors de son moi quelque autre idée intolérable. Ces malades aiment leur délire comme ils s'aiment eux-mêmes. Voilà tout le secret [de ces réactions]. ${ }^{13}$

Freud assimile donc le délire psychotique à une réaction psychique défensive possédant un aspect narcissique. II l'interprète sur le modèle du symptôme névrotique: le délire serait un retour du refoulé.

\subsection{Le manuscrit K (1896)}

En 1896, Freud postule que deux conditions sont nécessaires au déclenchement des psychonévroses: "II faut que l'incident provocateur ait été d'ordre sexuel et ensuite qu'il se soit produit avant la maturité sexuelle $" .{ }^{14}$ II en ajoute une troisième, relative à la dimension héréditaire, qu'il n'explicite guère. Selon lui, la dimension psychopathologique des psychonévroses provient d'un dérèglement de mécanismes psychiques normaux: " de conflits (dans l'hystérie), d'autoreproches (dans la névrose obsessionnelle), de rancune (dans la paranoïa), de deuil (dans le délire hallucinatoire aigu) $" .{ }^{15}$ Dans la paranoïa, la rancune se dérègle graduellement pour se transformer, passé un certain seuil, en méfiance. Elle en est le symptôme primaire. Dans ce manuscrit, Freud tente des rapprochements entre névrose et psychose sur la base, notamment, de l'argument suivant: l'éprouvé de l'incident primaire de la psychose serait analogue à celui de la névrose obsessionnelle, à savoir le plaisir. Si, dans la paranoïa, l'incident primaire est éprouvé avec plaisir, en revanche son souvenir engendre du "déplaisir dont le prochain est rendu responsable, suivant le mécanisme psychique de la projection ". ${ }^{16}$

1.4. Les nouvelles psychonévroses de défense (1896)

Deux ans après son premier article promouvant la nouvelle catégorie des psychonévroses, Freud estime que ses vues antérieures sont confirmées, en particulier celle consistant à "faire de la défense le point nucléaire dans le mécanisme psychique des névroses $"{ }^{17}$ 
Néanmoins, elles méritent d'être en partie amendées afin d'intégrer ses avancées afférentes aux manuscrits $\mathrm{H}$ et $\mathrm{K}$, parmi lesquelles figure le rattachement de la paranoïa au groupe des psychonévroses. Freud postule que chacune des psychonévroses se singularise tant sur le mécanisme de refoulement utilisé que sur le contenu du refoulé imprimant une forme particulière aux symptômes. ${ }^{18}$ Dans cette recherche, il s'aide de l'observation d'une femme de trente-deux ans présentant une paranoïa. Il s'engage alors dans la première cure psychanalytique d'un sujet psychotique ${ }^{19}$ en supposant que "des pensées inconscientes et des souvenirs refoulés [...] peuvent être amenés à la conscience lorsque est surmontée une certaine résistance $"{ }^{20}$ Freud trouve dans cette cure la confirmation de son présupposé. II recueille de la patiente ses pensées inconscientes et refoulées, notamment son souvenir le plus ancien, où elle se déshabillait devant son frère sans éprouver la moindre honte. Elle évoque également des attouchements sexuels. Freud considère que la patiente se reprochait aprèscoup, dans le présent, ces scènes de nudité réciproque avec son frère. Aussi, écrit-il, au sujet des hallucinations de la jeune femme au cours desquelles elle voyait des sexes féminins: "J'avais donc appris que ces hallucinations n'étaient rien d'autre que des fragments du contenu des expériences infantiles refoulées, des symptômes du retour du refoulé " ${ }^{21}{ }^{21}$ ॥ s'attache à analyser ces symptômes afin de dégager le mécanisme de refoulement distinctif de la paranoïa :

Dans la paranoïa, le reproche est refoulé sur une voie qu'on peut désigner comme projection, et le symptôme de défense qui est érigé est celui de la méfiance à l'égard des autres; la reconnaissance est ainsi refusée au reproche, et, comme par représailles, il n'existe aucune protection contre les reproches qui font retour dans les idées délirantes. $^{22}$

La projection permet de passer d'un autoreproche, à un reproche adressé à autrui, puis, à un reproche fait par autrui. On peut ainsi résumer l'approche freudienne de la formation du symptôme, caractérisée par le refoulement, dans chacune des trois psychonévroses : le lieu d'accrochage de l'affect, séparé de la représentation refoulée, se situe dans le corps pour l'hystérie, dans d'autres représentations substitutives pour la névrose obsessionnelle et dans l'autre pour la paranoïa.

\section{Le Président Schreber (1911)}

Jusqu'à cette époque, Freud peine à opérer une véritable clinique différentielle entre psychose et névrose, ayant peu exploité la notion de Verwerfung (1894). Son analyse des Mémoires d'un névropathe de D.-P. Schreber lui permet de faire un véritable saut conceptuel dans son approche de la psychose, en y introduisant le sujet. II considère la psychose déclarée de Schreber comme l'incidence clinique d'un "grave conflit psychique qui avait éclaté en lui $" .^{23}$ Dès lors, empruntant en partie les voies ouvertes par Karl Abraham ${ }^{24}$, Freud se livre à l'investigation de ce conflit, de ses causes actuelles et prédisposantes. Ce faisant, il met à jour certaines propriétés de la « structure de la paranoïa ${ }^{25} "$, laquelle est appréhendée à partir d'une stratification du psychisme et de son dynamisme.

\subsection{La période d'incubation}

Après une première hospitalisation, de décembre 1884 à juin 1885 dans la clinique du Pr. Flechsig, l'état psychique de Schreber se dégrade au cours du second semestre 1893. Il est de nouveau hospitalisé, cette fois-ci à l'asile de Sonnenstein. Commençant son analyse par la période d'incubation de ce second épisode, Freud relève deux faits importants survenus entre la nomination de Schreber à la présidence de la Cour suprême du Land de Dresde en juin 1893 et son entrée en fonction en octobre 1893. À l'époque le président rêve plusieurs fois du retour de sa pathologie où apparaît la figure du Pr. Flechsig, et il s'imagine la chose suivante: "ce doit être une chose singulièrement belle que d'être une femme en train de subir l'accouplement $»{ }^{26}$ Freud soupçonne qu'il 
existe un lien entre son attirance inconsciente pour Flechsig et cette rêverie homosexuelle. Ce fantasme de transformation en femme constitue l'élément primaire à partir duquel le délire va se construire. Un détail clinique confirme son hypothèse : lors d'une absence prolongée de sa femme, Schreber est en proie à un nouvel " effondrement nerveux ${ }^{27}$ consécutif, selon lui, à « un nombre tout à fait inaccoutumé de pollutions ". D'après Freud, cet effondrement apparaît sous l'influence conjointe de deux causes: des fantasmes homosexuels inconscients nécessaires aux multiples pollutions nocturnes et l'absence de son épouse qui exerçait jusquelà un rôle protecteur en empêchant son mari de succomber à son inclinaison inconsciente. Freud considère que la mort de son frère aîné, Gustav en 1877 , et celle de son père en 1861 s'accordent avec son hypothèse de la nostalgie fixée par transfert sur Flechsig. S'interrogeant sur les conditions d'émergence de cette nostalgie, Freud évoque les difficultés d'enfantement rencontrées par le couple Schreber et la frustration qui en découle. De telle sorte, écrit Freud, que :

\footnotetext{
Schreber peut très bien s'être imaginé que, s'il était une femme, il aurait mieux su s'y prendre pour avoir des enfants, et c'est ce qui lui ouvrit la voie de la régression jusqu'aux premières années de son enfance en lui permettant de se replacer dans l'attitude féminine envers son père qu'il avait eue alors. $^{28}$
}

Freud éclaire ainsi la régression du Président jusqu'à une position féminine centrale dans la construction de son délire.

\subsection{Les modes paranoïaques de contradiction du fantasme homosexuel}

Reconduisant sa thèse originelle des psychonévroses, Freud atteste qu' « aimer un homme, constitue le noyau du conflit dans la paranoïa de I'homme ". ${ }^{29}$ Puis, il se ravise, considérant que ce conflit psychique relatif à une homosexualité inconsciente refusée n'est nullement propre à la paranoïa, ni même à la psychose. Seuls seraient exclusivement paranoïaques trois modes de contradiction de l'énoncé « Moi (un homme) je l'aime (lui, un homme) $"{ }^{30}$ Chacun d'eux correspond à un délire particulier : « Le délire de jalousie contredit le sujet, le délire de persécution, le verbe, l'érotomanie, l'objet. " Si le postulat du délire de jalousie résulte directement de la contradiction du sujet de cet énoncé, en revanche les postulats des délires de persécution et érotomaniaque nécessitent une seconde étape, celle de la projection. Dans ces deux cas, la projection opérant sur le sujet de la contradiction, la proposition se trouve rejetée sur un autre. Freud en conclut que la projection représente le trait le plus marquant dans la formation des symptômes paranoïaques. II suppose que le lien entre le contenu de ce qui est réprimé et ce qui revient de l'extérieur s'apparente au refoulement, qui provient selon Freud d'une stratification en trois phases ${ }^{31}$ :

- le refoulement originaire assimilable à une fixation prédisposante ;

- le refoulement secondaire ou refoulement proprement dit, d'une époque plus récente ;

- le retour du refoulé qui actualise en quelque sorte cette fixation.

Freud a postulé jusque-là que la seconde strate du refoulement dans la paranoïa opère par la voie de la projection, le sujet projetant sur une ou plusieurs personnes un sentiment homosexuel qu'il réprime. Conscient que ce mécanisme est impropre à rendre compte de la paranoïa, il fait alors une avancée majeure dans son approche du phénomène psychotique en affirmant "que ce qui a été aboli au-dedans revient du dehors $»{ }^{32}$ Si le processus normal du refoulement secondaire correspond à un retrait de la libido investie sur un objet, Freud postule qu'intervient dans la paranoïa un détachement plus efficace nommé "détachement paranoïaque ". Ce faisant, il tente de cerner un mécanisme psychotique plus radical que le refoulement névrotique.

\section{Un cas de paranoïa chez une jeune femme (1915)}

En 1915, Freud rapporte une autre observation d'entrée dans la psychose. Embarrassé par l'une 
de ses clientes se plaignant de persécutions de la part de son ancien amant, un avocat initie une rencontre avec Freud à qui la jeune femme raconte son histoire. Courtisée depuis quelque temps par un collègue de travail, elle accepte un rendez-vous chez lui. Lors de leurs ébats, elle est surprise par un bruit "semblable à un battement ou à un tintement $"{ }^{33}$ À sa sortie, elle croise deux hommes qui semblent chuchoter sur son passage, l'un d'eux dissimulant un appareil photo. Se rappelant le bruit entendu dans la chambre, elle s'imagine que ce dernier a photographié leur intimité. Soucieuse, elle presse de questions son amant, mais non apaisée par ses réponses, elle contacte un avocat. Allant à première vue à l'encontre de sa conception de la paranoïa, Freud demande une seconde rencontre. La jeune femme revient sur sa première version et lui dit qu'en réalité, c'est au cours du deuxième rendez-vous qu'elle a été dérangée par le bruit sur lequel elle a ensuite greffé ses soupçons: on avait fomenté contre elle un piège visant à la compromettre. En outre, il apprend que le lendemain de leur premier rendez-vous, la jeune femme a vu à son travail son amant en conversation avec sa supérieure. En observant cette scène, la jeune femme a la certitude que son amant lui dévoile leur aventure amoureuse ou pire, qu'il entretient une relation amoureuse avec elle.

Selon Freud, cette dernière représente une figure maternelle et l'amant, en dépit de son jeune âge, une figure paternelle. II ramène ainsi la triade composée par la jeune femme, son amant et la supérieure aux conditions du complexe d'CEdipe.

Si la jeune femme est attirée par le substitut paternel, elle n'en reste pas moins sous la domination de son attachement à sa mère figurée ici par sa supérieure, pour laquelle elle éprouve des pulsions homo-sexuelles. Elle se trouve donc confrontée à un impossible, l'amour pour cet homme, que traite le délire: "Celui-ci était à l'origine dirigé [...] contre la femme, mais maintenant, sur le terrain de la paranoïa, était accompli le passage de la femme à l'homme comme objet $"{ }^{34}$
Grâce à cette observation, Freud trouve à confirmer ses thèses majeures: le sujet et son persécuteur sont de même sexe et le déclenchement de la paranoïa opère comme la mise en place d'une défense contre une homosexualité excessivement forte représentant «la disposition à l'affection paranoïaque $»{ }^{35}$

\section{Le cas d'un jeune médecin (1916-1917)}

Freud soutient en mai 1907 que le mécanisme de la projection paranoïaque doit être divisé en deux temps, à savoir «la décomposition en refoulement de la libido et en retour de la libido $"{ }^{36}$ Autrement dit, le sujet retire à une représentation d'objet son investissement libidinal qu'il transforme ensuite en une perception, avant de la projeter à l'extérieur. Le mois suivant, Freud construit trois cas de refoulement dont nous ne retenons que le deuxième, celui où "le refoulement se transforme en rejet [Verwerfung] » de la réalité. Conception d'ordre spéculatif, car comme il l'écrit à Jung peu après, la paranoïa " ne montre que le retour de la libido $"{ }^{37}$ Entre 1916 et 1917 , il suppose que, lors de ce retour de la libido, la tendresse initiale peut évoluer en haine. Cette dernière constitue dès lors un danger extrême voire vital pour l'objet, celui-ci étant simultanément aimé et haï. ${ }^{38} \|$ rapporte alors l'observation d'un jeune médecin ayant quitté sa ville d'exercice suite aux menaces de mort proférées à l'encontre de son meilleur ami, devenu l'objet de son délire de persécution. Lors de brefs entretiens, Freud apprend que les deux hommes se sont laissés aller à leur attirance réciproque. Puis, le jeune médecin lui confie que sa maladie s'est déclenchée lorsqu'il a réussi, pour la première fois, à satisfaire sexuellement une femme. II ressent alors une vive douleur au niveau du crâne qu'il compare à la sensation "qu'on éprouverait si on vous faisait sauter la boîte crânienne [...] ainsi qu'on le fait dans les autopsies ou les vastes trépanations ". Or, l'ami avec lequel il avait eu des relations intimes s'était justement spécialisé dans l'anatomie pathologique, branche de la 
médecine nécessitant l'ouverture des corps humains. Le jeune médecin conçut progressivement que cet ami lui avait fait connaître cette femme pour le tenter. Dès lors, il lui attribua l'ensemble des autres persécutions dont il était l'objet. Les éléments cliniques rapportés par Freud s'ordonnent selon une chronologie allant de la sensation primitive jusqu'à l'identification du persécuteur en la personne de l'amant. Cette temporalité implique chez Freud d'attribuer au délire paranoïaque la fonction d'une défense contre une poussée de libido homosexuelle.

\section{Un accès de jalousie délirante (1921)}

Dans son article de 1921, centré sur l'investigation psychanalytique des mécanismes psychiques de la jalousie, Freud discerne trois " strates ou stades de la jalousie:

1) concurrentielle ou normale 2) projetée 3) délirante $"{ }^{39}$ Dans la jalousie délirante, le sujet et son objet étant de même sexe, son développement nécessite une forte pulsion homosexuelle contre laquelle le sujet se défend à l'aide d'une contradiction de l'énoncé fantasmatique, "transcrite [chez l'homme] par la formule: Je ne l'aime pas, c'est elle qui l'aime $"{ }^{40}$ Dans cette perspective, Freud présente le cas d'un jeune homme présentant des accès de jalousie délirante qui

\footnotetext{
[...] apparaissaient régulièrement le lendemain d'un acte sexuel, au demeurant satisfaisant pour les deux parties. On est en droit de conclure, présume Freud, que chaque fois, après l'assouvissement de la libido hétérosexuelle, la composante homosexuelle excitée en même temps s'exprimait par la force dans la crise de jalousie. $^{41}$
}

Partant, un accès de jalousie délirante constitue chez ce sujet l'incidence clinique d'une satisfaction sexuelle. Puis, Freud ajoute que le sujet " n'avait formé ni amitié ni intérêts sociaux ", si bien que l'on " ne pouvait s'empêcher d'avoir l'impression que c'était comme si le délire n'avait eu qu'à assumer le développement ultérieur de ses relations avec l'homme, comme pour rattraper une part de ce qui avait été manqué $"{ }^{42}$ Freud attribue au délire une fonction permettant au sujet d'assumer ce qu'il avait d'abord manqué. De quoi s'agit-il ? Freud évoque, d'une part, l'absence du père et un lien très fort à la mère - il avait déjà souligné un tel lien chez la jeune femme de son observation de 1915 -, et d'autre part, l'existence d'un traumatisme homosexuel dans l'enfance du sujet, élément traumatique nodal dans sa doctrine des psychoses. Cette observation corrobore l'élaboration de Freud dans la mesure où le persécuteur était l'objet de même sexe le plus aimé du sujet.

\section{L'entrée dans la psychose et la seconde topique freudienne}

En 1924, Freud consacre deux articles à la promotion d'une clinique différentielle entre névrose et psychose. Leur différence porte davantage sur leur déclenchement respectif que dans "la tentative de réparation qui la suit ". ${ }^{43}$ II y envisage l'entrée dans la névrose comme le résultat d'un conflit opposant le ça au moi. En revanche, la psychose déclenchée s'enracine dans l'ouverture $d$ ' " une faille dans la relation du moi au monde extérieur $"{ }^{44}$ équivalant à une perte de la réalité. La seconde topique permet à Freud de distinguer, à partir de relations à trois termes (le moi, le ça et la réalité), les éclosions des névroses, de celles des psychoses. Si l'agent reste le même, à savoir le moi, l'opération et la nature de ce sur quoi elle porte diffèrent. Dans le cas de la psychose, Freud précise que le retrait du moi d'un fragment de la réalité s'effectue au profit du ça. Reprenant le cas de $M^{\text {elle }}$ Élisabeth von $R^{45}$, il décline les deux réactions possibles, névrotique et psychotique, devant la situation présentée comme suit : une jeune femme, au chevet du lit de mort de sa sœur, se dit à propos de son beau-frère, dont elle est amoureuse, que maintenant qu'il est libre, il peut l'épouser. La réaction névrotique, indique Freud, a été de refouler ce désir qui a trouvé à se satisfaire et à se dire dans les symptômes hystériques, alors que «la réaction 
psychotique aurait été de dénier [Verleugnen] le fait de la mort de la sœur $"{ }^{46}$ Dénier un tel événement représente pour Freud la perte d'un morceau de réalité à laquelle succède un second temps. En effet, à l'instar de la névrose, il discerne deux temps dans la psychose: "Le premier coupant le moi [...] de la réalité, le second, en revanche, essayant de réparer les dégâts et reconstituant aux frais du ça la relation à la réalité. " Néanmoins, et la différence est cruciale, la névrose ne procède pas d'un déni de réalité. Puis en 1937, Freud estime que l'entrée dans la psychose est consécutive à un déni actuel d'un morceau de réalité, qui actualise un premier déni survenu dans l'enfance. Cela signifie que la représentation déniée était psychiquement conservée et non abolie. De surcroît, poursuit-il, le délire puise son pouvoir de conviction dans la "vérité historique qu'il met à la place de la réalité repoussée $"{ }^{47}$ II en conclut, qu'à l'instar de l'hystérique, le psychotique souffre de réminiscences. Devant sa trouvaille de la vérité historique du déni, Freud n'a pas d'autre modèle que celui de la névrose pour construire celui de la psychose: le mécanisme du refoulement est pour lui conceptuellement indépassable. II ne réussit jamais à élever au statut théorique certaines de ses indications relatives au rejet ou Verwerfung portant sur une représentation unique.

\section{Conclusion : la quête freudienne}

Pour conclure, soulignons que l'approche freudienne de l'entrée dans la psychose repose sur un modèle causal classique nécessitant deux implications : une prédisposition psychotique et un événement devant lequel le sujet réagit grâce à un mécanisme de défense identique au mécanisme psychique déterminant la structure psychotique.
La psychose freudienne se particularise donc par une structure dont le déclenchement, "un Ausbruch, un éclatement de la psychose, comme le soulignait déjà Freud dès le Manuscrit $H$ de 1895 " intervient " devant un événement repérable comme déclencheur. ${ }^{48}$

Cependant, ce mécanisme reste obscur, Freud ne parvenant pas à dégager ses propriétés distinctives. Freud lui-même

\footnotetext{
[...] semble avoir de plus en plus douté, comme il le confie en 1915, que le processus nommé refoulement dans la schizophrénie ait quoi que ce soit de commun avec le refoulement dans les névroses de transfert, c'est pourquoi il s'efforça de dégager la spécificité d'un mécanisme psychotique. Le concept de "Verleugnung", plus rarement celui de "Verwerfung", furent les principaux utilisés à cette fin. ${ }^{49}$
}

Dans les années vingt, Freud avance le concept de Verleugnung traduit par déni, démenti ou désaveu, après celui de Verwerfung habituellement traduit par rejet dont les premières occurrences datent de 1894.

Si Freud ne put concevoir un mécanisme original de la psychose, il en jeta les bases nécessaires ayant " défriché le terrain en cernant ce point resté vacant dans la théorie psychanalytique dont, par la suite, la notion de forclusion forgée par Lacan rendra raison $"{ }^{50}$

Aussi «la quête freudienne d'un mécanisme spécifique ${ }^{51}$ de la psychose mérite d'être distinguée d'autant qu'elle ne fut jamais interrompue : dans son Abrégé de psychanalyse, le fondateur de la psychanalyse cherchait encore "les conditions nécessaires à l'apparition d'une psychose $» .{ }^{52}$

Elle incite à reconnaître ce que les élaborations ultérieures des psychanalystes doivent aux découvertes inaugurales et singulières de Freud. 


\section{Bibliographie :}

Aparicio, S. (1984). La forclusion, préhistoire d'un concept. Ornicar ? Revue du Champ freudien, 28, 83.

Freud, S. (1911). Remarques psychanalytiques sur l'autobiographie d'un cas de paranoïa (Le Président Schreber). Cinq psychanalyses. Paris : PUF.

Freud, S. (1961). Introduction à la psychanalyse (19161917). Paris : Payot.

Freud, S. (1969). La naissance de la psychanalyse. Paris: PUF.

Freud, S. (1975). Abrégé de psychanalyse (1938). Paris: PUF.

Freud, S. (1985). Sur les types d'entrée dans la névrose (1912). Névrose, psychose et perversion. Paris : PUF.

Freud, S. (1995). Constructions dans l'analyse (1937). Résultats, idées, problèmes, II, 1921-1938. Paris : PUF.

Freud, S. (2002). Mademoiselle Élisabeth v. R.... In Breuer, J. \& Freud, S. Études sur l'hystérie (1895). Paris : PUF.

Freud, S. \& Abraham, K. (2006). Correspondance complète, 1907-1925, Paris : Gallimard.

Freud, S. \& Jung, C. G. (1975). Correspondance, I, 19061909. Lettre 25F, datée du 23 mai 1907. Paris : Gallimard. Hoffmann, C. (2004). Quelques réflexions à propos du déclenchement de la psychose et de ses suppléances dans le monde de l'adolescent contemporain. Figures de la psychanalyse, 9.

Maleval, J.-C. (1993). Construction et évolution du concept de Forclusion du Nom-du-Père. Les Cahiers de Cliniques Psychologiques, université Rennes 2, 17.

Maleval, J.-C. (2000). La forclusion du Nom-du-Père. Paris : Seuil.

Schreber, D.-P. (1975). Mémoires d'un névropathe (1903). Paris : Seuil.

\section{Notes :}

${ }^{1}$ Cet article constitue une version allégée d'un chapitre de notre ouvrage L'entrée dans la psychose (2011) paru aux Presses universitaires de Rennes.

${ }^{2}$ Freud, S. (1985). Sur les types d'entrée dans la névrose (1912). Névrose, psychose et perversion. Paris : PUF.

${ }^{3}$ Freud, S. (1894). Les psychonévroses de défense. Névrose, psychose et perversion. Op. cit., p. 13.

${ }^{4}$ Ibid., p. 12.

${ }^{5}$ Maleval, J.-C. (2000). La forclusion du Nom-du-Père. Paris : Seuil, p. 39.

${ }^{6}$ Freud, S. (1894). Les psychonévroses de défense. Op. cit., p. 13.

${ }^{7}$ Ibid., p. 12.

${ }^{8}$ Ibid., p. 13.

${ }^{9}$ Freud, S. (1969). La naissance de la psychanalyse. Paris : PUF, p. 98.

${ }^{10}$ lbid., p. 99.
${ }^{11}$ lbid., p. 99-100.

${ }^{12}$ Ibid., p. 100.

${ }^{13}$ Ibid., p. 101

${ }^{14}$ Ibid., p. 130.

${ }^{15}$ Ibid., p. $129-130$.

${ }^{16}$ Ibid., p. 135.

${ }^{17}$ Freud, S. (1896). Nouvelles remarques sur les psychonévroses de défense. Névrose, psychose et perversion. Op. cit., p. 61.

${ }^{18}$ Ibid., p. 72.

${ }^{19}$ Maleval, J.-C. (2000). La forclusion du Nom-du-Père. Op. cit., p. 375.

${ }^{20}$ Freud, S. (1896). Nouvelles remarques sur les psychonévroses de défense. Op. cit., p. 74.

${ }^{21}$ lbid., p. 77-78.

${ }^{22}$ Ibid., p. 80.

${ }^{23}$ Freud, S. (1911). Remarques psychanalytiques sur l'autobiographie d'un cas de paranoïa (Le Président Schreber). Cinq psychanalyses. Paris : PUF, p. 281.

${ }^{24} \mathrm{Cf}$. Les lettres 96F, 98F et 110F. Freud, S. \& Abraham K. (2006). Correspondance complète, 1907-1925, Paris: Gallimard.

${ }^{25}$ Freud, S. (1911). Remarques psychanalytiques sur l'autobiographie d'un cas de paranoïa. Op. cit., p. 263.

${ }^{26}$ Schreber, D.-P (1975). Mémoires d'un névropathe (1903). Paris : Seuil, p. 46.

${ }^{27}$ Schreber cité par Freud. Ibid., p. 293.

${ }^{28}$ Ibid., p. 304.

${ }^{29}$ Ibid., p. 308.

${ }^{30}$ Ibid., p. 309. II en ajoute un quatrième, opérant dans le délire des grandeurs qui rejette entièrement la proposition : Je n'aime que moi.

${ }^{31}$ Ibid., p. 311.

${ }^{32}$ Ibid., p. 315.

${ }^{33}$ Freud, S. (1915). Communication d'un cas de paranoïa en contradiction avec la théorie psychanalytique. Névrose, psychose et perversion. Op. cit., p. 210.

${ }^{34}$ Ibid., p. 217.

${ }^{35}$ Ibid., p. 216.

${ }^{36}$ Freud, S. \& Jung C. G (1975). Correspondance, I, 1906-1909. Lettre 25F, datée du 23 mai 1907. Paris : Gallimard, p. 95.

${ }^{37}$ Ibid., p. 111-112. Lettre 30F, datée du 6 juin 1907.

${ }^{38}$ Freud, S. (1961). Introduction à la psychanalyse (19161917). Paris : Payot, p. 402.

${ }^{39}$ Freud, S. (1922). Sur quelques mécanismes névrotiques dans la jalousie, la paranoïa et l'homosexualité. Névrose, psychose et perversion. Op. cit., p. 271.

${ }^{40}$ lbid., p. 273.

${ }^{41}$ lbid., p. 274.

${ }^{42}$ Ibid., p. 275.

${ }^{43}$ Freud, S. (1924). La perte de la réalité dans la névrose et dans la psychose. Névrose, psychose et perversion. Op. cit., p. 301. 
${ }^{44}$ Freud, S. (1924). Névrose et psychose. Névrose, psychose et perversion. Op. cit., p. 285.

${ }^{45}$ Freud, S. (2002). Mademoiselle Élisabeth v. R.... in Breuer, J. \& Freud, S. Études sur l'hystérie (1895). Paris : PUF.

${ }^{46}$ Freud, S. (1924). La perte de la réalité dans la névrose et dans la psychose. Op. cit., p. 300.

${ }^{47}$ Freud, S. (1995). Constructions dans l'analyse (1937). Résultats, idées, problèmes, II, 1921-1938, Paris: PUF, p. 280.

${ }^{48}$ Hoffmann, C. (2004). Quelques réflexions à propos du déclenchement de la psychose et de ses suppléances dans le monde de l'adolescent contemporain. Figures de la psychanalyse, 9, p. 50.

${ }^{49}$ Maleval, J.-C. (1993). Construction et évolution du concept de Forclusion du Nom-du-Père. Les Cahiers de Cliniques Psychologiques, université Rennes 2, 17, p. 47.

${ }^{50}$ Aparicio, S (1984). La forclusion, préhistoire d'un concept. Ornicar ? revue du Champ freudien, 28, p. 83.

${ }^{51}$ Maleval, J.-C. (2000). La forclusion du Nom-du-Père. Op. cit., p. 34.

${ }^{52}$ Freud, S. (1975). Abrégé de psychanalyse (1938). Paris: PUF, p. 78.

\section{L'auteur :}

\section{Yohan Trichet}

Docteur en psychologie, membre associé du Laboratoire Recherches en psychopathologie: nouveaux symptômes et lien social (EA 4050), UFR Sciences Humaines, Université Rennes 2

Place du recteur Henri Le Moal

Bâtiment S - CS 24307

\section{Rennes Cedex}

France.

\section{Référence électronique}

Yohan Trichet, "La clinique freudienne de l'entrée dans la psychose", Recherches en Psychanalyse [En ligne], 12|2011, mis en ligne le 22 décembre 2011.

Texte intégral

Droits d'auteur

Tous droits réservés 